\section{Treatment of pelvic organ prolapse}

\author{
Munir'deen A. ljaiya, Hadijat O. Raji \\ Department of Obstetrics and \\ Gynaecology, University of Ilorin \\ Teaching Hospital, llorin, Nigeria
}

\begin{abstract}
Prolapse of the pelvic organs is a common condition encountered in gynecological practice that adversely affects the quality of life of affected women. It affects millions of women worldwide. The principles of treatment of pelvic organ prolapse include restoring anatomy and vaginal function, correcting associated urinary and or fecal incontinence, and preventing de novo prolapse and incontinence. There are various treatment options for pelvic organ prolapse. These vary from conservative treatments/mechanical interventions to surgery. The choice of treatment depends on severity of symptoms, patient's age, parity, and whether there is the need to conserve the uterus for reproductive function. In conclusion, thorough evaluation of symptoms and degree of prolapse is essential in order to provide the best possible treatment and ultimately improve quality of life.
\end{abstract}

\section{Introduction}

Pelvic organ prolapse occurs when there is loss of support to the structures within the pelvis resulting in a downward displacement of these structures together with the vagina. Organs that may be involved include the bladder, urethra, uterus, small bowel or rectum, causing a urethrocoele, cystocoele, cystourethrocoele, enterocoele, rectocoele, uterovaginal prolapse, apical prolapse or various combinations of these. Sometimes there is vault prolapse following hysterectomy.

The first occurrence of pelvic organ prolapse was reported in $2000 \mathrm{BC}$ and various treatments were described for it. Hippocrates described numerous non-surgical treatments for pelvic organ prolapse. ${ }^{1}$ In $98 \mathrm{AD}$, Soranus of Rome first described the removal of the prolapsed uterus when it became black. The first successful vaginal hysterectomy was described by Willouby in 1670 when Faith Raworth performed the procedure on herself. ${ }^{1}$ She was a peasant woman who was so debilitated by uterine prolapse that she pulled down on the cervix and slashed off the prolapse with a sharp knife. She did, however, suffer complications of hemorrhage which she survived, and also devel- oped urinary incontinence. ${ }^{1}$

Pelvic organ prolapse is a condition that affects millions of women worldwide; ${ }^{2}$ the exact incidence and prevalence is, however, unknown since epidemiological studies determining this are rare. ${ }^{3}$ Studies have quoted an incidence of approximately 3-9\% of adult women. ${ }^{4,5}$ It is one of the most common indications for gynecological surgery in developed countries. In the US, it is estimated that over 1 billion dollars is spent annually on the treatment of this condition and it is listed as the third most common indication listed for hysterectomy in all women and the most common indication for hysterectomy in menopausal women. ${ }^{6,7}$

Several factors contribute to the occurrence of uterovaginal prolapse. Factors that have been cited as increasing the likelihood of a woman developing uterovaginal prolapse include advancing age, pelvic trauma from vaginal delivery, instrumental vaginal delivery and hereditary factors. Other factors that may contribute include lifting of heavy weights, degenerative changes as a result of withdrawal of estrogen support at menopause, bearing down against an incompletely dilated cervix, chronically raised intraabdominal pressure from chronic cough or chronic constipation, and connective tissue disorders. However, vaginal delivery and increasing age are said to be the most common factors. ${ }^{8}$

It has been said that rather than a single factor, it is more probable that combinations of anatomical, physiological, genetic, lifestyle and reproductive factors interact throughout a woman's life span to contribute to pelvic floor disorders. ${ }^{9}$ The risk of prolapse increases 4 fold after the birth of the first child and this increases 11-fold after four or more deliveries. ${ }^{9}$ Prolapse is also more common among Caucasians and Asians than Africans. In the Women's Health Initiative, $41 \%$ of women aged 50-79 years showed some amount of pelvic organ prolapse, including cystocoele in $34 \%$, rectocoele in $19 \%$, and uterine prolapse in $14 \% .^{10}$

Pelvic organ prolapse may, however, be asymptomatic and even when symptoms are present no correlation has been found between the degree of prolapse and their severity. Symptoms reported by patients with prolapse include feeling a fullness in the vagina, feeling something coming down in the vagina, noticing a protrusion in the vagina, and also urinary and bowel symptoms such as, among others, urinary frequency, stress incontinence, difficulty in defecation, or having to manually reduce the mass before defecation. Some women complain about a dragging sensation, and even low back pain and problematic sexual intercourse.
Correspondence: Munir'deen A. Ijaiya, Department of Obstetrics and Gynaecology, University of Ilorin Teaching Hospital, PMB 1339, Ilorin, Nigeria.

Tel. $+2348033801565-+2348057068400$.

E-mail: munirijaiya@yahoo.com

Key words: pelvic organ, prolapse, treatment.

Received for publication: 19 August 2011.

Revision received: 9 January 2012.

Accepted for publication: 8 February 2012.

This work is licensed under a Creative Commons Attribution NonCommercial 3.0 License (CC BYNC 3.0).

(C) Copyright M. A. Ijaiya, H.O. Raji, 2012

Licensee PAGEPress, Italy

Urogynaecologia 2012; 26:e3

doi:10.4081/uij.2012.e3

\section{Treatment}

\section{Treatment of asymptomatic pelvic organ prolapse}

In cases of asymptomatic pelvic organ prolapse, watchful expectancy is recommended. This involves examining the patient periodically to detect any progression of the prolapse, as well as asking about any symptom which may just be developing. Patients should also be advised on weight reduction, dietary modification and pelvic floor exercises.

\section{Treatment of symptomatic pelvic organ prolapse}

Intervention is usually indicated if the patient is symptomatic; this may or not involve surgery. Choice of treatment modality usually depends on the type and size of the prolapse, the organs contained within the prolapse, presence and severity of symptoms, patient age, desire for future sexual intercourse and fertility, as well as presence of other medical conditions. Prior to surgery, women should be evaluated for stress urinary incontinence and, if present, may have a concomitant anti-incontinence surgery. In women without stress incontinence, latent incontinence may become apparent following prolapse repair.

\section{Non-surgical management}

Non-surgical management includes conservative management [(lifestyle changes such as losing weight, dietary modification to prevent constipation, treatment of chronic diseases such as chronic cough, delivering pelvic floor muscle training PFMT)], estrogen therapy, as well as the use of mechanical interventions like pessaries. The use of electrical stim- 
ulation is no longer recommended because it has been shown to be ineffective.

A Cochrane database systematic review has shown some evidence to indicate a positive effect of pelvic floor muscle training for prolapse symptoms and severity. Six months of supervised PFMT has benefits in terms of improved anatomical and symptoms (if symptomatic) immediately post-intervention. ${ }^{11}$ This is supported by the pelvic organ prolapsed physiotherapy (POPPY) multicenter trial, which suggested that PFMT delivered by a physiotherapist to symptomatic mild pelvic organ prolapsed women in an outpatient setting may reduce the prolapse severity. ${ }^{12}$ However, no further evidence of the efficacy and cost-effectiveness of PFMT for symptomatic prolapse in the medium and long term is available. ${ }^{11}$

Pessaries are one of the oldest remedies for prolapse. ${ }^{8}$ Some recent studies have shown that pessaries are effective in alleviating symptoms and patient satisfaction is high..$^{13}$ According to the 2004 Cochrane database systematic review, there was no evidence from randomized controlled trials upon which to base treatment of women with pelvic organ prolapse through the use of different types of device, define the indications, nor guide the pattern of replacement and follow-up care. ${ }^{14}$ These may be offered to women who have symptomatic prolapse, elderly women who are poor candidates for surgery, those who wish to defer surgery to possibly complete their family, for short-term relief before surgery, or to offer long-term relief for women who do not want to undergo surgery. ${ }^{14,15}$ However, these are not supported by definitive evidence. ${ }^{14}$ Pessaries can also be used during pregnancy. They have been found to alleviate most of the symptoms of prolapse including voiding symptoms, urgency-related symptoms, bowel symptoms and sexual dysfunction.

Pessaries come in various shapes and sizes, and are usually more beneficial for patients with a lesser degree of prolapse. Gelhorn pessary is ideal for advanced prolapse. ${ }^{16}$ Evidence for pessary selection and management is incomplete and is based on a trial and error approach, expert opinion and experience..$^{14,17}$ The pessary is placed in the vagina above the pelvic floor musculature. It presses against the vaginal walls and is retained within the vagina by the tissues of the vaginal outlet. The pessary remains in place during ambulation; the patient should not be aware of it when in situ and the pessary should remain in place during micturition. Pessaries are fitted by the doctor and the patient should also be taught how to remove, clean and reinsert it.

Factors to be considered when fitting a pessary include the size of the vagina, the nature and extent of prolapse, and the desire for sexual activity. The appropriate size and shape of the pessary should be inserted such that the prolapse is effectively reduced and the woman is comfortable with the device in place. The doctor should be able to sweep his or her finger between the pessary and the walls of the vagina. The patient should be asked to undertake various activities, including standing, walking, performing a Valsalva maneuver, and bending, to ensure that the pessary is retained. She should also be able to void without difficulty with the pessary in place before leaving the clinic. ${ }^{18}$ Complications include vaginal discharge, discomfort, bleeding, and in neglected cases, vaginal ulceration and fistulas. ${ }^{15}$

\section{Surgical management of prolapsed pelvic organ}

The aim of surgery is to re-suspend the prolapsed organs thereby returning them to their normal position, to reinforce the pelvic supports, and to improve the woman's quality of life. Pelvic organ prolapse surgery may be a reconstructive or obliterative surgical procedure. The types of surgical repair vary depending on the types of prolapse and associated symptoms. The impact of the surgery on bladder, sexual and bowel function cannot be predicted and may make symptoms worse or result in new symptoms, such as leakage of urine or problematic intercourse. ${ }^{19}$

\section{Reconstructive surgery}

Approaches to reconstructive surgery for uterovaginal prolapse could be vaginal, abdominal, laparoscopic or via combined procedures. For successful repair, it is imperative to have identified the extent and structures involved in the prolapse and to choose the correct surgical approach. This is said to be critical for surgery to be successful. An active search for potential and undeveloped defects must be made at the time of prolapse surgery to reduce the chance of failure. ${ }^{20}$ Site-specific repair of pelvic support defects is currently advocated..$^{21}$ This helps to maximally restore the normal biochemical support and suspension of the central pelvic organs.

Anterior colporrhaphy is the surgical treatment of choice for anterior vaginal wall prolapse. It involves dissection of the vaginal mucosa through a midline incision of the anterior vagina in order to expose the underlying bladder and pubocervical fascia, which is then repaired by placation; the redundant vaginal tissue is excised, and closed in the midline. The durability of this traditional approximation of native tissue is in question; therefore, synthetic meshes have become a popular adjunct to provide additional support. Randomized controlled trials have shown that anterior colporrhaphy with reinforced vaginal paravaginal repair using xenograft or synthetic mesh in women with symptomatic anterior vaginal wall prolapse have a lower anatomical failure rate on examination than standard anterior colporrhaphy. ${ }^{19,22}$ But no difference has been seen in terms of quality of life data, de novo dyspareunia, stress incontinence, reoperation rates for prolapse or incontinence. Mesh erosion was reported in $10 \%$ of anterior repairs with polypropylene mesh..$^{19}$ Use of a trochar-guided mesh kit has a higher rate of surgical complications and post-operative adverse events. ${ }^{23}$ Other complications associated with graft use in transvaginal pelvic organ prolapse repair include hemorrhage, visceral injury and fistula. ${ }^{24}$

Posterior colporrhaphy is the surgical management of rectocoele and limited evidence suggests that vaginal surgery may be better than transanal surgery for rectocoele. ${ }^{19}$ Posterior colporrhaphy and anterior colporrhaphy are usually referred to as pelvic floor repair. A systematic review of graft use in transvaginal pelvic organ prolapse repair carried out by Sung et al. of the Society of Gynecologic Surgeons systematic review group showed that the use of graft in posterior vaginal wall repair is not superior to native tissue repair for anatomic or symptomatic outcomes. $^{24}$

\section{Non-uterus sparing surgical proce- dure}

Vaginal hysterectomy is frequently performed for uterine prolapse. It is the traditional and definitive treatment of uterine prolapse. Vaginal hysterectomy alone without an anterior or posterior repair does not improve pelvic support. In this procedure, once the uterus has been removed, the vaginal apex should be attached to a point that is higher in the pelvis than the vagina in order to elevate it to a higher position; this is critical for a successful repair. ${ }^{8}$ Anterior colporrhaphy is performed for patients with cystocoele and a posterior colporrhaphy if a rectocoele is present.

\section{Uterus sparing surgical procedures}

Uterus sparing pelvic reconstructive surgeries are indicated in young women who need to retain the uterus for reproductive function, such as women who have not completed their family. i) Manchester repair is one of the earlier surgical reconstructive options for the management of uterovaginal prolapse. It was described by Archibald Donald of Manchester in 1888 as a feasible alternative option to vaginal hysterectomy for treatment of uterovaginal prolapse. ${ }^{20}$ The procedure involves amputation of the cervix, shortening and approximation of the cardinal ligament, and anterior colporrhaphy with or without posterior colporrhaphy. Manchester repair has not proved to be so popular in recent times because of the challenge to fertility that results from amputation of the 
cervix. It is associated with shorter operating time, less blood loss, and lower morbidity and mortality than vaginal hysterectomy. ${ }^{20}$ ii) Sacrohysteropexy/sacrocervicopexy is performed by inserting a mesh anteriorly to the posterior lower uterus or junction of the cervix and uterus, connecting it to anterior longitudinal ligaments over the first sacral vertebra. This is useful in correcting uterine prolapse. iii) Transvaginal uterosacral suspension/application was described in a small group of young women who had uterovaginal prolapse in $1966 .{ }^{20}$ The procedure involved performing posterior colpotomy to assess the pouch of Douglas. The uterosacral ligaments were cut, plicated and re-inserted into the cervix. The same procedure was carried out for the cervical ligament; this was later plicated across the midline of the anterior surface of the cervix. The simpler method of Uterosacral ligament vaginal vault suspension may be performed either vaginally or abdominally. It involves attaching the vaginal apex to remnants of the uterosacral ligaments at the level of the ischial spines or higher. iv) Sacrospinous ligament fixation (sacrospinous hysteropexy) is indicated for apical prolapse. It involves the suspension of the sacrospinous ligaments unilaterally or bilaterally using a vaginal extraperitoneal approach. Recurrent apical prolapse is said to be uncommon following this procedure, ${ }^{6}$ vaginal depth, axis and function are restored, and enterocoele formation is prevented by uterosacral plication. The challenge of reduced fertility and the dyspareunia associated with some other uterus conserving surgical procedures are not experienced with this method..$^{20}$ v) Abdominal sacral colpopexy involves the suspension of the vaginal vault to the sacral promontory. It can also be used to correct high cystocoele and rectocoele with a single mesh in an inverted $\mathrm{Y}$ shaped fashion. The mesh is inserted anteriorly to correct a high cystocoele and posteriorly down to the perineal body to correct a rectocoele. ${ }^{15}$ Among the advantages of this procedure is that it conserves the normal vaginal anatomy and prevents shortening of the vagina. A major complication is hemorrhage from the pre-sacral vessels.

The results of a Cochrane review of forty randomized controlled trials showed that abdominal sacral colpopexy was associated with a lower rate of recurrent vaginal vault prolapse and dyspareunia than with vaginal sacrospinous colpopexy. However, it has a longer operating time, longer recovery time before return to daily routine activities, and higher cost of surgery than vaginal sacrospinous colpopexy. However, there was no statistically significant difference in reoperation rates for prolapse..$^{19}$ vi) Infracoccygeal sacropexy or posterior intravaginal slingplasty is another method of managing uterine or vaginal vault prolapse. A study by De
Tayrac et al. showed that infracoccygeal sacropexy has similar prolapse cure rates, symptom scores and quality of life to sacrospinous suspension. It is faster and easier to perform, and less painful than sacrospinous suspension. ${ }^{25}$ vii) In pectineal ligament suspension, the uterus is suspended to the pectineal ligament bilaterally with a merselene tape. This technique is a simple, safe and effective treatment for uterine prolapse in young women. ${ }^{20}$

\section{Laparoscopic procedures}

With the advent of minimally invasive surgeries, many cases of uterovaginal prolapse can now be managed through a laparoscopic route. Procedures like vaginal hysterectomy, uterosacral suspension, and hysteropexy can be performed successfully laparoscopically.

\section{Treatment of enterocoele or vault repair}

Some of the enterocoeles can be dealt with during vaginal hysterectomy for uterine prolapse. Extensive posterior colpoperineorrhaphy or sacrospinous colpopexy are used to repair enterocoeles that occur with or without vault prolapse after abdominal or vaginal hysterectomy. Many of the enterocoeles can be repaired during abdominal surgery by coaptation of the uterosacral ligaments. The procedure described by Moschowitz has been discouraged because of the risk of injury to the ureter while applying the purse string sutures into the pouch of Douglas peritoneum and there is little inherent strength in the peritoneum. ${ }^{15,26,27}$ Other possible treatment options include iliococcygeal fixation and infracoccygeal sacropexy or posterior intravaginal slingplasty which is performed to correct an enterocoele or vaginal vault suspension complicating hysterectomy.

Most gynecologists prefer transabdominal sacrocolpopexy for recurrent prolapse repair. A mesh that is peritonealized is sutured to the vault and then attached to the anterior longitudinal ligament over the first or second sacral vertebra. ${ }^{15,26}$

There is no consensus among surgeons as to which suture material should be used for repair of pelvic organ prolapse. Some advocate the use of non-absorbable suture as they liken prolapse repair to a series of herniorrhaphies and non-absorbable sutures are used for hernia repair. Others, however, use delayed absorbable suture according to the view that the suture material is no longer needed once healing is complete.

Up to $40 \%$ of patients have been found to have a recurrence in pelvic organ prolapse following surgery. ${ }^{28}$ This may be attributed to an underlying connective tissue defect in the pathogenesis of pelvic organ prolapse. ${ }^{29}$
Because of this high percentage of women who will need a second surgical intervention for pelvic organ prolapse, there has been a continuous effort to improve surgical procedures and outcomes. This has led to the use of biological and synthetic mesh for surgical treatment.,28,29 Over the last decade, mesh has become popular in pelvic reconstructive surgery. There have been some improvements in mesh development to reduce the complications and adverse effects, such as production of lightweight mesh with less dense and larger pores. This prevents the mesh wrinkling and folding, and ensures pliability after surgery. Medical manufacturers have produced a pelvic floor replacement system that restores the support structures. The anterior system consists of a central mesh portion and two lateral arms on each side that are placed through the obturator foramina about $4 \mathrm{~cm}$ apart. The posterior kit has a central mesh portion with arms that go through the buttock, traverse the ischiorectal fossa, and enter the pelvis via the iliococygeus muscle or sacrospinous ligament. ${ }^{30}$ Prolift is an example of such a surgical kit system. It is made up of anatomical guides, retrieval devices, partially absorbable mesh and implant. Perigee and Apogee are anterior and posterior mesh systems, respectively. Others include Avaulta, Elevate and Surelift. Recently, in the United States of America, intravaginal mesh surgeries have generated a lot of controversy because of the attendant adverse effects. The United States Food and Drug Administration carried out a systematic review of the scientific literature to learn more about the safety and effectiveness of pelvic organ prolapse and stress urinary incontinence using surgical mesh. This revealed that adverse events are not rare, contrary to what had been stated in the 2008 Public Health Notification. ${ }^{31}$

The common complications of transvaginally placed mesh include erosion, dyspareunia, infection, urinary problems, bleeding and organ perforation. Other complications are recurrent prolapse, re-operation, mesh contraction (vaginal scarring and shrinkage) and death. The increase in the number of serious adverse effects is a cause for concern. However, abdominal pelvic organ prolapse surgery using mesh (sacral colpopexy) appears to result in lower rates of mesh complications compared to transvaginal pelvic organ prolapse surgery with mesh..$^{30}$ Therefore, mesh surgery should only be chosen after weighing the risks and benefits of surgery with mesh versus all surgical and non-surgical alternatives, and patients should be adequately counseled.

Apart from synthetic meshes, different types of grafts have also been used to improve support of the pelvic organs during reconstructive surgery. Autologous grafts are harvested from another area of the body e.g. rectus abdominis or fascia lata; complications include hematoma and a weakened fascia at harvest 
site. Allografts come from a human source other than the patient and may include cadaveric fascia or cadaveric dermis; to avoid the complication of vaginal erosion, some surgeons use cadaveric fascia instead of synthetic mesh, but this is associated with a higher rate of prolapse recurrence. ${ }^{32}$ Xenografts are biological tissue obtained from a specie foreign to the patient, e.g. porcine dermis or bovine pericardium. Biological materials are, however, associated with a higher rate of recurrence, ${ }^{2}$ possibly due to absorption of these materials by the host tissue. ${ }^{29}$

\section{Obliterative procedures}

Obliterative procedures may be offered to elderly women who are no longer sexually active and are poor anesthetic risks, or women with medical conditions, which preclude surgery in which coitus is no longer desired. They involve removal of extensive vaginal epithelium and suturing the anterior and posterior vaginal walls together. This results in obliteration of the vaginal vault and effective occlusion of the vagina. This procedure includes partial colpocleisis or LeFort's operation and complete colpocleisis. The difference is that in Le Fort's colpocleisis, rectangular portions of the vaginal mucosa are dissected from the anterior and posterior vaginal walls and then apposed together to occlude the vaginal vault. Lateral tracts are created on either side of the closed vagina and these serve as drainage channels. In complete colpocleisis on the other hand, the entire vaginal wall is excised. ${ }^{2}$ Both procedures can only be performed if the woman does not wish to be sexually active.

\section{Conclusions}

It is important to remember that prolapse is essentially a quality of life issue. As such, complete evaluation of patients' symptoms, and assessment of the extent and degree of prolapse is essential in order to offer the patient the best management possible, alleviate her symptoms and improve her quality of life.

\section{References}

1. Lazarou G, Grigorescu BA. Pelvic organ prolapse. Available from: http//emedicine.medscape.com/article/276259-overview

2. Schorge J0, Schaffer JI, Halvorson LM, et al. (eds) Pelvic organ prolapse. In: Williams gynaecology. New York: McGraw-Hill Publishers; 2008; pp. 532-555.

3. Bump RC, Norton PA. Epidemiology and natural history of pelvic floor dysfunction. Obstet Gynecol Clin North Am 1998;25:723-46.
4. Slieker-ten HMCP, Vierhout M, Bloembergen H, Schoenmaker G. Distribution of pelvic organ prolapse in a general population: prevalence, severity, etiology, and relation with the function of pelvic floor muscles. Abstract presented at the Joint Meeting of the ICS and IUGA, Aug 25-27, 2004, Paris, France.

5. Swift SE, Woodman P, O'Boyle A, et al. Pelvic organ support study (POSST); the distribution clinical definition and epidemiologic condition of pelvic organ support defects. Am J Obstet Gynecol 2005;192:795-806.

6. Pearce M, Swift S, Goodnight W. Pelvic organ prolapse: is there a difference in $\mathrm{POPQ}$ exam results based on time of day, morning or afternoon? Am J Obstet Gynecol 2008;199:200.el-5.

7. Wilcox LA, Koonin LM, Pokras R, et al. Hysterectomy in the United States, 19881990. Obstet Gynecol 1994;83:549-55.

8. DeLancey JOL, Strohbehn K. Pelvic organ prolapse. In: JR Scott, RS Gibbs, BY Karlan, AF Haney (eds). Danforth's obstetrics and gynaecology. Philadelphia: Lippincott Williams and Wilkins; 2003. pp. 791-817.

9. DeLancey JOL, Kane Low L, Miller JM, et al. Graphic integration of causal factors of pelvic floor disorders: an integrated life span model. Am J Obstet Gynecol 2008; 199:610.e1-5.

10. Hendrix SL, Clark A, Nygaard I, et al. Pelvic organ prolapse in the WOMEN'S Health Initiative: gravity and gravidity. Am J Obstet Gynecol 2002;186:1160-6.

11. Hagen S, Stark D. Conservative prevention and management of pelvic organ prolapse in women. Cochrane Database Syst Rev 2011; 12:CD003882.

12. Hagen S, Stark D, Glazener C, et al. A randomized controlled trial of pelvic floor muscle training for stages I and II pelvic organ prolapse. Int Urogynecol J Pelvic Floor Dysfunct 2009;20:45-51.

13. Lamers BHC, Broekman BMW, Milani AL. Pessary treatment for pelvic organ prolapse and health-related quality of life: a review. Int Urogynecol J Pelvic Floor Dysfunct 2011; 22:637-44.

14. Adams E, Thomson A, Maher C, Hagen S. Mechanical devices for pelvic organ prolapse in women. Cochrane Database Syst Rev 2004:CD004010.

15. Vimplis S, Hooper P. Assessment and management of pelvic organ prolapse. Curr Obstet Gynaecol 2005;15:387-93.

16. Biller D, Davila GW. Management of genital prolapse. In: G Gheniem, W Davila (eds). Practical guide to female pelvic medicine. 1st ed. London: Taylor and Francis; 2006. pp. 16982.

17. Atnip SD. Pessary use and management for pelvic organ prolapse. Obstet Gynecol Clin North Am 2009;36:541-63.

18. Jelovsek JE, Maher C, Barber MD. Pelvic organ prolapse. Lancet 2007;369:1027-8.
19. Maher C, Feiner B, Baessier K, Glazener CMA. Surgical management of pelvic organ prolapse in women. Cochrane Database Syst Rev 2010;4:CD004014.

20. Diwan A, Rardin CR, Kohli N. Uterine preservation during surgery for uterovaginal prolapse: a review. Int Urogynecol J Pelvic Floor Dysfunct 2004;15:286-92.

21. Zimmerman CW. Pelvic organ prolapse: Basic Principles. In: JA Rock, HW Jones 3rd (eds.). TeLinde's operative gynecology. 10th ed. Philadelphia: Lippincott Williams and Wilkins; 2008. pp. 854-73.

22. Menefee SA, Dyer KY, Lukacz ES, et al. Colporrhaphy compared with mesh or graftreinforced vaginal repair for anterior vaginal wall prolapse: a randomized controlled trial. Obstet Gynecol 2011;118: 1337-44.

23. Altman D, Vayrynen T, Engh ME, et al. Anterior colporrhaphy versus transvaginal mesh for pelvic-organ prolapse. $\mathrm{N}$ Engl J Med 2011;364:1826-36.

24. Sung VW, Rogers RG, Schaffer JT, et al. Graft use in transvaginal pelvic organ prolapse repair. A systematic review. Obstet Gynecol 2008;112:1131-42.

25. De Tayrac R, Mathe ML, Bader G, et al. Infracoccygeal sacropxy or sacrospinous suspension for uterine or vaginal vault prolapse. Int J Gynecol Obstet 2008;100:154-9.

26. Stanton SL. Vaginal prolapse. In: DK Edmond (ed.). Dewhurst's textbook of obstetrics and gynaecology for postgraduates. 6th ed. London: Blackwell Science; 2000. pp. 462-473.

27. Hudson C, Setchell ME (eds). Operations for genital prolapse and uterine displacements. In: RW Shaw. Shaw's textbook of operative gynaecology. 6th ed. New Delhi, India: Elsevier, 2004. pp. 200-222.

28. Martan A. New options in reconstructive pelvic floor surgery and surgery in urogynecology. Ceska Gynecol 2006;71;6;455-63.

29. Stanton SL. Vaginal prolapse. In: RW Shaw, WP Soutter, SL Stanton (eds.). Gynaecology. 3rd ed. Edinburgh: Churchill Livingstone; 2003. pp. 813-824.

30. Jeffery S, De Jong P. Mesh, grafts and kits in pelvic organ prolapse surgery: where are we now? South Afr Urol Gynaecol Rev 2009; 6:1322.

31. Urogynecologic surgical mesh: update on the safety and effectiveness of transvaginal placement for pelvic organ prolapse. Centre for Devices and Radiological Health, Food and Drugs Administration. July 2011, pp 3-15. Available from: http:/www.fda.gov/downloads/medical devices/safety/alerts and notices/UCM 262760.pdf

32. Gregory WT, Otto LN, Bergstrom JO, Clark AL. Surgical outcome of abdominal sacrocolpopexy with synthetic mesh versus sacrocolpopexy with cadaveric fascia lata. Int Urogynecol J Pelvic Floor Dysfunct 2005;16; $369-74$. 